$$
\begin{gathered}
a=1, \\
b, c, d,>K
\end{gathered}
$$

and such that the conclusion of Theorem 1 is false.

Proof. Let $b=N, d=2^{\dot{M}}-2, c=2^{-r}(d !)$, where $r$ is chosen to make $c$ an odd integer. Clearly $a$ is $P_{s}(b)$ only for $s=1$. Now $c$ is not $P_{1}(d)$, provided $d>3$, and not $P_{2}(d)$ since an odd integer cannot be the sum of two odd integers. Hence, we cannot find partitions of $a, c$ satisfying the conclusions of Theorem 1.

Suppose $d$ is $P_{s}(c)$. Then

$$
d=d_{1}+d_{2}+\cdots+d_{s}
$$

where each $d_{i} \leqq d$ and $\left(d_{i}, c\right)=1$. Now $c$ is divisible by all odd integers $<d$; therefore $d_{i}$ is a power of 2 . I.e.,

$$
d=2^{r_{1}}+2^{r_{2}}+\cdots 2^{r_{s}} .
$$

Since $d=2^{M}-2$ there are at least $M-1$ summands in (14). I.e., if $d=P_{s}(c)$, then $s \geqq M-1$. But clearly if $b$ is $P_{s}(a)$, then $s \leqq N$. If we now choose $M-1>$ $N+1$ and $M, N$ large enough to ensure $b, c, d>K$, the conclusion of Theorem 2 follows.

International Business Machines Corporation

Thomas J. Watson Research Center

Yorktown Heights, New York

1. C. C. Elgot \& J. D. Rutledge, "Operations on Finite Automata," Proceedings of the Second Symposium on Switching Circuit Theory and Logical Design (AIEE: 5-134), October, 1961.

\title{
Approximations to Kelvin Functions
}

\section{By F. D. Burgoyne}

While preparing a digital computer program to examine the behavior of largetaper hub flanges, it was found necessary to use approximations to the Kelvin functions ber $x$, bei $x$, $\operatorname{ker} x$, and kei $x$, and to their first derivatives. To obtain full machine accuracy, the approximations were required to be correct to nine significant figures. Several tabulations of these functions exist, but the only ones considered to be sufficiently accurate were those of Lowell [1] and Nosova [2]; however, limitations of internal memory in the computer used precluded the possibility of storing such tables and interpolating.

The functions actually required were $Z_{i}(x)$ and $Z_{i}^{\prime}(x)(1 \leqq i \leqq 4)$, where

$$
\begin{aligned}
& Z_{1}(x)=\text { ber } x \\
& Z_{2}(x)=- \text { bei } x \\
& Z_{3}(x)=-\frac{2}{\pi} \operatorname{kei} x \\
& Z_{4}(x)=-\frac{2}{\pi} \operatorname{ker} x ;
\end{aligned}
$$

Received Feburary 5, 1962. 
and the approximations below, which were obtained by Lanczos' economization procedure [3], enable these functions to be evaluated for all positive $x$, correct to at least nine significant figures.

$$
\begin{aligned}
& Z_{1}(10 t)=c_{10}+c_{11} t^{4}+\cdots+c_{18} f^{32} \quad\left(\max . \text { error } 0 \leqq t \leqq 1, \quad 27 \times 10^{-13}\right) \\
& t^{-2} Z_{2}(10 t)=c_{20}+c_{21} t^{4}+\cdots+c_{28} t^{32} \quad\left(\text { “ “ “ “ } 2 \times 10^{-13}\right) \\
& t^{-2} R_{1}(10 t)=c_{30}+c_{31} t^{4}+\cdots+c_{38} t^{32} \quad\left(\text { " " " } \quad \text { " } 7 \times 10^{-13}\right. \text { ) } \\
& t^{-4} R_{2}(10 t)=c_{40}+c_{41} t^{4}+\cdots+c_{48} t^{32} \quad\left(\text { “ “ “ “ } 1 \times 10^{-13}\right. \text { ) } \\
& t^{-3} Z_{1}^{\prime}(10 t)=d_{10}+d_{11} t^{4}+\cdots+d_{18} t^{32} \quad\left(\text { " “ “ “ } \text { “ } 1 \times 10^{-13}\right) \\
& t^{-1} Z_{2}^{\prime}(10 t)=d_{20}+d_{21} t^{4}+\cdots+d_{28} t^{32} \quad\left(\text { " " " } \quad \text { " } 7 \times 10^{-13}\right) \\
& t^{-1} R_{1}^{\prime}(10 t)=d_{30}+d_{31} t^{4}+\cdots+d_{38} t^{32} \quad\left(\text { " “ " } \text { " } \quad 25 \times 10^{-13}\right. \text { ) } \\
& t^{-3} R_{2}{ }^{\prime}(10 t)=d_{40}+d_{41} t^{4}+\cdots+d_{48} t^{32} \quad\left(\text { " “ “ } \text { “ } 2 \times 10^{-13}\right. \text { ) } \\
& S(10 / t)=e_{10}+e_{11} t+\cdots+e_{18} t^{8} \quad\left(\max . \text { error }-1 \leqq t \leqq 1,5 \times 10^{-10}\right) \\
& T(10 / t)=e_{20}+e_{21} t+\cdots+e_{28} t^{8} \quad\left(\text { " “ } \text { " } \quad \text { " } 5 \times 10^{-10}\right) \\
& U(10 / t)=e_{30}+e_{31} t+\cdots+e_{38} t^{8} \quad\left(\text { “ “ } \text { " } \quad \text { “ } 5 \times 10^{-10}\right) \\
& V(10 / t)=e_{40}+e_{41} t+\cdots+e_{48} t^{8} \quad\left(\text { " " } \text { " } \quad \text { " } 5 \times 10^{-10}\right) \text {. }
\end{aligned}
$$

TABLE 1

\begin{tabular}{l|r|r|r|r}
\hline$i$ & \multicolumn{1}{c|}{$c_{1 \mathrm{i}}$} & \multicolumn{1}{c|}{$c_{2 \mathrm{i}}$} & \multicolumn{1}{c}{$c_{3 \mathrm{i}}$} & \multicolumn{1}{c}{$c_{4 \mathrm{i}}$} \\
\hline 0 & +0.9999999999974 & -24.9999999999998 & +24.9999999999993 & +234.3750000000000 \\
1 & -156.2499999995701 & +434.0277777777479 & -795.7175925924866 & -1412.8508391203636 \\
2 & +678.1684027663091 & -678.1684027769807 & +1548.4845196730992 & +1153.8281852814561 \\
3 & -470.9502795889968 & +240.2807549442574 & -623.0136717405201 & -255.0971742710479 \\
4 & +93.8596692971726 & -28.9690338786499 & +81.9524771606200 & +21.2123451660231 \\
5 & -7.2422567278207 & +1.4963342749742 & -4.5187459132639 & -0.8061529027876 \\
6 & +0.2597773000700 & -0.0384288282734 & +0.1222087382192 & +0.0159380149705 \\
7 & -0.0048987125727 & +0.0005444243175 & -0.0018064777860 & -0.0001797627986 \\
8 & +0.0000516070465 & -0.0000044913000 & +0.0000154363047 & +0.0000012161109 \\
\hline
\end{tabular}

TABLE 2

\begin{tabular}{r|r|r|r|r}
\hline$i$ & \multicolumn{1}{|c|}{$d_{1 \mathrm{i}}$} & \multicolumn{1}{c|}{$d_{2 \mathrm{i}}$} & \multicolumn{1}{c}{$d_{3 \mathrm{i}}$} & \multicolumn{1}{c}{$d_{4 \mathrm{i}}$} \\
\hline 0 & -62.4999999999999 & -4.9999999999993 & +4.9999999999975 & +93.7499999999998 \\
1 & +542.5347222222147 & +260.4166666665533 & -477.4305555551536 & -1130.2806712962694 \\
2 & -565.1403356479486 & -678.1684027747539 & +1548.4845196652035 & +1384.5938223372452 \\
3 & +150.1754718432278 & +336.3930569023651 & -872.2191403672455 & -408.1554788292578 \\
4 & -14.4845169498403 & -52.1442608975905 & +147.5144585913337 & +42.4246903131088 \\
5 & +0.6234726348243 & +3.2919352108579 & -9.9412403209725 & -1.9347669229237 \\
6 & -0.0137246036190 & -0.0999147064932 & +0.3177418434686 & +0.0446263862145 \\
7 & +0.0001701453451 & +0.0016331100837 & -0.0054188558408 & -0.0005752042283 \\
8 & -0.0000012506046 & -0.0000152269884 & +0.0000523294314 & +0.0000043682053 \\
\hline
\end{tabular}


Table 3

\begin{tabular}{c|c|c|c|c}
$i$ & $e_{1 i}$ & $e_{2 i}$ & $e_{3 i}$ & $e_{4 i}$ \\
\hline 0 & +1.0000000000 & +0.0000000000 & +1.0000000000 & +0.0000000000 \\
1 & +0.0088388346 & -0.0088388340 & -0.0265165040 & +0.0265165034 \\
2 & +0.0000000007 & -0.0007031241 & -0.0000000008 & +0.0011718740 \\
3 & -0.0000517869 & -0.0000518006 & +0.0000725024 & +0.0000725179 \\
4 & -0.0000112207 & -0.0000000072 & +0.0000144255 & +0.0000000079 \\
5 & -0.0000016192 & +0.0000016431 & +0.0000019780 & -0.0000020042 \\
6 & +0.0000000135 & +0.0000005929 & -0.0000000147 & -0.0000006992 \\
7 & +0.0000001452 & +0.0000000750 & -0.0000001671 & -0.0000000883 \\
8 & +0.0000000492 & -0.0000000243 & -0.0000000563 & +0.0000000269 \\
\hline
\end{tabular}

$$
\begin{aligned}
& Z_{3}(x)=\frac{1}{2} Z_{1}(x)-\frac{2}{\pi}\left\{R_{1}(x)+Z_{2}(x) \log \left(\frac{1}{2} x e^{\gamma}\right)\right\} \\
& Z_{4}(x)=\frac{1}{2} Z_{2}(x)+\frac{2}{\pi}\left\{R_{2}(x)+Z_{1}(x) \log \left(\frac{1}{2} x e^{\gamma}\right)\right\} \\
& Z_{3}^{\prime}(x)=\frac{1}{2} Z_{1}^{\prime}(x)-\frac{2}{\pi}\left\{R_{1}^{\prime}(x)+Z_{2}^{\prime}(x) \log \left(\frac{1}{2} x e^{\gamma}\right)+Z_{2}(x) / x\right\} \\
& Z_{4}^{\prime}(x)=\frac{1}{2} Z_{2}^{\prime}(x)+\frac{2}{\pi}\left\{R_{2}{ }^{\prime}(x)+Z_{1}^{\prime}(x) \log \left(\frac{1}{2} x e^{\gamma}\right)+Z_{1}(x) / x\right\}
\end{aligned}
$$

( $\gamma$ being Euler's constant $0.5772 \cdots$ ).

$$
\begin{aligned}
& Z_{1}(x)=(2 \pi x)^{-\frac{1}{2}} \exp \left(x / 2^{\frac{1}{2}}\right) \\
& \cdot\left\{S(x) \quad \cos \left(x / 2^{\frac{1}{2}}-\pi / 8\right)-T(x) \quad \sin \left(x / 2^{\frac{1}{2}}-\pi / 8\right)\right\} \\
& Z_{2}(x)=-(2 \pi x)^{-\frac{1}{2}} \exp \left(x / 2^{\frac{1}{2}}\right) \\
& \cdot\left\{T(x) \quad \cos \left(x / 2^{\frac{1}{2}}-\pi / 8\right)+S(x) \quad \sin \left(x / 2^{\frac{1}{2}}-\pi / 8\right)\right\} \\
& Z_{1}^{\prime}(x)=(2 \pi x)^{-\frac{1}{2}} \exp \left(x / 2^{\frac{1}{2}}\right) \\
& \left\{U(x) \quad \cos \left(x / 2^{\frac{1}{2}}+\pi / 8\right)-V(x) \quad \sin \left(x / 2^{\frac{1}{2}}+\pi / 8\right)\right\} \\
& Z_{2}^{\prime}(x)=-(2 \pi x)^{-\frac{1}{2}} \exp \left(x / 2^{\frac{1}{2}}\right) \\
& \cdot\left\{V(x) \quad \cos \left(x / 2^{\frac{1}{2}}+\pi / 8\right)+U(x) \quad \sin \left(x / 2^{\frac{1}{2}}+\pi / 8\right)\right\} \\
& Z_{3}(x)=-(2 / \pi x)^{\frac{1}{2}} \exp \left(-x / 2^{\frac{1}{2}}\right) \\
& \cdot\left\{T(-\mathrm{x}) \cos \left(x / 2^{\frac{1}{2}}+\pi / 8\right)-S(-x) \sin \left(x / 2^{\frac{1}{2}}+\pi / 8\right)\right\} \\
& Z_{4}(x)=-(2 / \pi x)^{\frac{1}{2}} \exp \left(-x / 2^{\frac{1}{2}}\right) \\
& \cdot\left\{S(-x) \cos \left(x / 2^{\frac{1}{2}}+\pi / 8\right)+T(-x) \sin \left(x / 2^{\frac{1}{2}}+\pi / 8\right)\right\} \\
& Z_{3}{ }^{\prime}(x)=(2 / \pi x)^{\frac{1}{2}} \exp \left(-x / 2^{\frac{1}{2}}\right)
\end{aligned}
$$$$
\cdot\left\{V(-x) \cos \left(x / 2^{\frac{1}{2}}-\pi / 8\right)-U(-x) \sin \left(x / 2^{\frac{1}{2}}-\pi / 8\right)\right\}
$$ 


$$
\begin{aligned}
Z_{4}{ }^{\prime}(x)=(2 / \pi x)^{\frac{1}{2}} \exp \left(-x / 2^{\frac{1}{2}}\right) & \\
\cdot & \cdot\left\{(-x) \cos \left(x / 2^{\frac{1}{2}}-\pi / 8\right)+V(-x) \sin \left(x / 2^{\frac{1}{2}}-\pi / 8\right)\right\} .
\end{aligned}
$$

The constants $c_{i j}, d_{i j}$, and $e_{i j}(1 \leqq i \leqq t, 0 \leqq j \leqq 8)$ are given in Tables 1 to 3 .

Battersea College of Technology

London, S.W.11.

1. H. H. Lowell, Tables of the Bessel-Kelvin Functions Ber, Bei, Ker, Kei, and their Derivatives for the Argument Range 0(0.01)107.50, Technical Report R-32, National Aeronautics and Space Administration, Washington, D. C., 1959.

2. L. N. Nosova, Tables of Thomson Functions and their First Derivatives, Izdatel'stvo AkademiY Nauk SSSR, Moscow, 1960.

3. National Bureau of Standards Applied Mathematics Series, v. 9, Tables of Chebyshev Polynomials $S_{n}(x)$ and $C_{n}(x)$, U.S. Government Printing Office, Washington, 1952.

\section{Quadrature Formulas over Infinite Intervals in Terms of Differences}

\section{By F. D. Burgoyne}

This paper describes quadrature formulas which may be used instead of GaussLaguerre or Gauss-Hermite formulas and in which differences are employed. The relative merits of each type of formula are considered and simple examples are given. While the idea behind them is certainly not new, the formulas themselves do not appear to have been previously published.

An integral of the type $\int_{0}^{\infty} e^{-x} f(x) d x$ if often evaluated numerically by means of a Gauss-Lgguerre quadrature formula. Thus we have

$$
\int_{0}^{\infty} e^{-x} f(x) d x=\sum_{i=0}^{n} H_{n i} f\left(a_{n i}\right)+E,
$$

where the weights $H_{n i}$ and the abscissas $a_{n i}$ have been extensively tabulated (see, for example, [1]), and $E$ represents an error term. Similarly, an integral of the type $\int_{-\infty}^{\infty} e^{-x^{2}} f(x) d x$ can be evaluated numerically by a Gauss-Hermite quadrature formula, and we have

$$
\int_{-\infty}^{\infty} e^{-x^{2}} f(x) d x=\sum_{i=0}^{n} K_{n i} f\left(b_{n i}\right)+F,
$$

where the $K_{n i}$ and the $b_{n i}$ have also been the subject of several tabulations (one of the most comprehensive being [2]), and $F$ is the error term. The advantage of such formulas is that for a given value of $n$ they attain the maximum possible degree of precision, i.e. $2 n+1$, and on this account they are usually very accurate. Further, if an automatic computer is being used, it may be convenient not to have to form differences.

Unfortunately they also suffer from several drawbacks. Perhaps the most serious is that having arrived at an estimate for the value of an integral using a certain value of $n$, if we then decide to use a different value of $n$ we have to repeat the entire calculation with new weights and abscissas. Although this is undesirable

Received September 24, 1962. 